Pacific Journal of Mathematics

LEVEL SETS ON SPHERES

LeE Meyers SONNEBorn 


\section{LEVEL SETS ON SPHERES}

\section{M. SONNEBORN}

The purpose of this paper is to prove that corresponding to any continuous real-valued function whose domain is the $n$-dimensional sphere $(n \geqq 2)$, there is a connected set on the sphere which contains a pair of antipodal points and on which the function is constant. While this constant need not be unique, a stronger property is found which ensures uniqueness and gives continuity to the constant over homotopies of the function.

The weaker theorem was stated in abstract by R. D. Johnson, Jr. [2]. The proof which follows constitutes a portion of the author's dissertation, [4].

Throughout this paper, $n$ will be used to denote any integer not less than 2. The usual $n$-dimensional measure on the $n$-sphere will be taken to be normalized so that the total measure of the sphere is one. Each time the measure of a set is mentioned, the set will be either open or closed, and therefore measurable. Everytime the components of a set are listed, the set will be open, and will therefore have a countable number of components. A subset of $S^{n}(n \geqq 2)$ will be said to be "too big" if it has measure greater than one-half. A subset of the sphere is said to "cut up" the sphere if no component of its complement is too big.

The fundamental tool to be used here is the following:

TheOREm. If $O$ is an open set on the n-sphere, then either $O$ or its complement $S^{n}-O$ has a component which cuts up the sphere.

The method of proof is to assume that $O$ has no such component and to prove that then its complement does.

Lemma 1. If $A$ is a connected subset of $S^{n}(n>1)$, and if $B$ is a component of $S^{n}-A$, then $S^{n}-B$ is connected, and $F(B)$, the boundary of $B$, is also connected.

Proof. $S^{n}$ is connected. The connectedness of $S^{n}-B$ follows from [3], page 78. $F(B)$ is connected since $F(B)=\bar{B} \cap \overline{S^{n}-B}$ and since $S^{n}$ is unicoherent. See [5] pages 47-60.

Henceforth, let $O$ denote an open subset of $S^{n}$, no component of which cuts up $S^{n}$. Corresponding to any component, $O_{i}$, of $O$, there is, then, a (unique-consider the measure) component, $T_{i}$, of its component, $S^{n}-O_{i}$, which is too big.

Received January 11, 1960, and in revised form July 18, 1960. 
LEMma 2. For $i \neq j$, either

(i ) $T_{i} \subseteq T_{j}$

(ii) $T_{j} \subseteq T_{i}$

(iii) $T_{i} \cup T_{j}=S^{n}$.

Proof. By Lemma 1, $T_{i}, T_{j}, S^{n}-T_{j}$, and $S^{n}-T_{i}$ are all connected as is the boundary of each. $O_{i}$ and $O_{j}$ are connected and disjoint. Each lies in a single component of the complement of the component of the other. Hence, either $O_{i} \subseteq T_{j}$ or $O_{i} \subseteq S^{n}-T_{j}$. In the first case, $S^{n}-O_{i}$ contains the connected set $S^{n}-T_{j}$, and $S^{n}-T_{j}$ is contained in a component of $S^{n}-O_{i}$. Either this component is $T_{i}$ or not. If it is $T_{i}$, then $S^{n}-T_{j} \subseteq T_{i}$ and $T_{i} \cup T_{j}=S^{n}$. If not, then $S_{n}-T_{j} \cap T_{i}=\phi$, and $T_{i} \subseteq T_{j}$. In the second case $O_{i} \subseteq S^{n}-T_{j}$ and $T_{j} \subseteq S^{n}-O_{i}$, so that $T_{j}$ being connected lies in a single component of $S^{n}-O_{i}$. But this component must be $T_{i}$, for it is the only one big enough to contain $T_{j}$ which is also too big. In this case, then $T_{j} \subseteq T_{i}$.

Corollary. For $i \neq j$, either

(i ) $S^{n}-T_{i} \supseteq S^{n}-T_{j}$

(ii) $S^{n}-T_{j} \supseteqq S^{n}-T_{i}$

(iii) $\left(S^{n}-T_{i}\right) \cap\left(S^{n}-T_{j}\right)=\phi$.

Now, let $O^{\prime}=\bigcup_{j}\left(S^{n}-T_{j}\right)$. Clearly $O^{\prime} \supseteqq O$ since for each $i, O_{i} \leqq$ $S^{n}-T_{i}$, and the $O_{i}$ are the components of $O . O^{\prime}$ is the union of open sets and is, therefore, open. Let $X_{j}, j=1,2, \cdots$ (possibly finite) be the components of $O^{\prime}$. Since for any $i, S^{n}-T_{i}$ is connected, it must lie entirely in one of the $X_{j}^{\prime}$ 's, and any $X_{j}$ is the union of all the $S^{n}-T_{i}$ 's contained in it.

LEMmA 3. If $S^{n}-T_{i}$ and $S^{n}-T_{j}$ are disjoint but are both contained in the same component, $Y_{k}$, of $O^{\prime}$, then there is an integer $l$ such that $S^{n}-T_{i} \leqq S^{n}-T_{l}$ and $S^{n}-T_{j} \subseteq S^{n}-T_{l}$.

Proof. Assume there is no such integer $l$. Let $T$ be the union of all $S^{n}-T_{m}$ which contain $S^{n}-T_{i}$. Clearly none of these intersects $S^{n}-T_{j}$ by the corollary to Lemma 2 . Let $S$ be an arc in $X_{k}$ connecting $x \in S^{n}-T_{i}$ to $y \in S^{n}-T_{j}$. ( $X_{k}$ is open and connected and hence arcwise connected). $\quad S$ must intersect $F(T)$. Let $p \in S \cap F(T) . \quad p \in S^{n}-T_{q}$ for some $q$ such that $S^{n}-T_{q} \in X_{k}$. Some neighborhood of $p$ also is in $S^{n}-T_{q}$ which is open. But this neighborhood of $p$ contains a point $z \in T$ since $p \in F(T)$. Hence $z \in S^{n}-T_{m}$ for some $m$ such that $S^{n}-$ $T_{m} \supseteqq S^{n}-T_{i}$. Since $S^{n}-T_{m}$ and $S^{n}-T_{q}$ intersect, one contains the other by the corollary to Lemma 2 . In either case, however, $S^{n}-T_{q} \subseteq$ $T$. But then $p \in T, p$ being a boundary point of the open set $T$. 
This contradiction establishes the lemma.

LEMMA 4. Each $Y_{k}$ can be written as a countable expanding union of sets $S^{n}-T_{j}$ (i.e. a union in which each set contains the previous).

Proof. If $X_{k}$ contains only a finite number of $S^{n}-T_{i}$, it contains a biggest one (repeated application of Lemma 3.) Suppose then, that $X_{k}=\bigcup_{i=1}^{\infty}\left(S^{n}-T_{i}\right)$. We choose a subunion of this union as follows: Let $I_{1}=S^{n}-T_{1}$; for $m>1$, let $L_{m}=S^{n}-T_{i(m)}$ where $i(m)$ is the smallest number for which $S^{n}-T_{i(m)} \supseteq S^{n}-T_{i(m-1)}$ if there is such a number $i(m)$. If, at some stage, there is no such number, the union will be finite; otherwise it will be countably infinite. It remains to be shown that $\bigcup_{m}\left(I_{m}\right)=X_{k}$. Let $x \in X_{k}$. If $x \in I_{1}, x \in \mathbf{U}_{m}\left(I_{m}\right)$, so suppose $x \notin I_{1} . \quad x \in S^{n}-T_{p}$ for some $p$. There is, therefore, a smallest integer $q$ for which $x \in S^{n}-T_{q}$ and $S^{n}-T_{q} \supseteqq I_{1}$ (Lemma 3). There is a largest integer $s$ for which $s=i(h)$ for some $h$, and $s<q$. It follows that $q=i(h+1)$ for $\left(S^{n}-T_{q}\right) \cap\left(S^{n}-T_{s}\right) \neq \phi$ and $x \in S^{n}-T_{q}$ while $x \notin S^{n}-T_{s}$. Hence $x \in \bigcup_{m}\left(I_{m}\right)$.

Lemma 5. For each $k$, the measure of $X_{k}$ does not exceed one-half.

Proof. Each $T_{i}$ has measure greater than one-half, so that each $S^{n}-T_{i}$ has measure less than one-half. The expanding union of open sets measuring less than one-half cannot have measure greater than one-half. [1].

LeMMA 6. $S^{n}-O^{\prime}$ is connected.

Proof. Each $S^{n}-X_{i}$ is either one of the $T_{j}$, or is expressible as the decreasing intersection of a countable number which are closed and connected. By Lemma 3.8 of page 80 of Wilder [5], $S^{n}-X_{i}$ is connected. Since $X_{i}$ is also connected, it follows from Lemma 1 that $F\left(X_{i}\right)$ is connected. Now suppose $S^{n}-O^{\prime}$ is not connected. Then $S^{n}-O^{\prime}=A \cup B$ where $A$ and $B$ are disjoint, nonempty, and relatively closed in $S^{n}-O^{\prime}$ and hence closed in $S^{n}$. Since each $X_{i}$ has a connected boundary, each $X_{i}$ has its boundary entirely in $A$ or entirely in $B$. Then consider $S^{n}=A^{\prime} \cup B^{\prime}$ where $A^{\prime}=A \cup\left(\bigcup_{i \in I} X_{i}\right), I=\left\{i \mid F\left(X_{i}\right) \subseteq A\right\}$ and $B^{\prime}=B \cup\left(\bigcup_{j \in J} X_{j}\right), J=\left\{j \mid F\left(X_{j}\right) \subseteq B\right) . \quad A^{\prime}$ and $B^{\prime}$ are easily seen to be closed, nonempty and disjoint. Hence $S^{n}$ is not connected. This contradiction establishes the lemma.

THEOREM 1. If $O$ is an open set of $S^{n}(n>1)$, then either $O$ or $S^{n}-O$ has a component which cuts up the sphere $(i, e$. a component 
whose complement consists of components with measures no than greater one-half).

Proof. All the previous lemmas except the first were based on the assumption that $O$ had no such component. Since $O^{\prime} \supseteqq O$ it follows that $S^{n}-O^{\prime} \subseteq S^{n}-O$. But $S^{n}-O^{\prime}$ is connected and lies in a component, $A$, of $S^{n}-O$. Since $A \supseteqq S^{n}-O^{\prime}, S^{n}-A \subseteq O^{\prime}$, and every component of $S^{n}-A$ is contained in a component of $O^{\prime}$. But the components of $O^{\prime}$ all have measure no greater than one-half, and so also do the components of $S^{n}-A$.

LEMma 7. If $A$ and $B$ are both connected, closed sets on $S^{n}$ which cut up $S^{n}$, then $A \cap B$ is not empty.

Proof. Suppose $A$ and $B$ are disjoint. $A$ being connected, lies in a single component, say $B_{1}$, of $S^{n}-B . S^{n}-B_{1}$ is connected (Lemma 1) and lies in a single component, say $A_{1}$ of $S^{n}-A$. Now the measure of the open set $A_{1}$ is strictly greater than the measure of the closed set $S^{n}-B_{1}$ contained in it. However, $M\left(B_{1}\right)<1 / 2$ by assumption, so that $M\left(S^{n}-B_{1}\right) \geqq 1 / 2$ and $M\left(A_{1}\right)>1 / 2$ contrary to the assumption that $A$ cuts up $S^{n}$. This contradiction establishes the theorem.

COROLLARY. If $g: S^{n} \rightarrow S^{n}$ is a measure-preserving homeomorphism, and if $A$ is a connected, closed subset of $S^{n}$ which cuts up $S^{n}$, then there is a point $x \in A$ for which $g(x) \in A$. In particular, any such set $A$, contains a pair of antipodal points of $S^{n}$.

THEOREM 2. Let $F: S^{n} \times I \rightarrow E^{1}$, be continuous, $(n>1)$, and define $f_{t}: S^{n} \rightarrow E^{1}$ for each $t, O \leqq t \leqq 1$, by $f_{t}(x)=F(x, t)$ for each $x \in S^{n}$. Then for each $t, O \leqq t \leqq 1$, there exists an unique real number $k_{t}$ such that $f_{t}^{-1}\left(k_{t}\right)$ contains a closed connected subset which cuts up $S^{n}$. This subset contains a pair of antipodal points of $S^{n}$. Further, $k_{t}$ is a continuous function of $t$ on $O \leqq t \leqq 1$.

Proof. The uniqueness of $k_{t}$ and the fact that the subset contains a pair of antipodal points follow from Lemma 7 and its corollary. The continuity of $k_{t}$ follows in the usual way from the compactness of $S^{n} \times I$ and the resulting uniform continuity of $F$. The existence of $k_{t}$ remains to be proved, that is it must be shown that for every function $f: S^{n} \rightarrow E^{1}$, there exists a real number $k$, such that $f^{-1}(k)$ contains a closed connected subset which cuts up $S^{n}$. For each positive integer $m$, there exists an open subset, $O_{m}$ of $E^{1}$ with the property that all components of both $O_{m}$ and of $E^{1}-O_{m}$ have diameter less than $1 / m$. For each $m, f^{-1}\left(O_{m}\right)$ is an open subset of $S^{n}$, so that according 
to Theorem 1, there is a component of either $f^{-1}\left(O_{m}\right)$ or of $S^{n}-$ $f^{-1}\left(O_{m}\right)=f^{-1}\left(E^{1}-O_{m}\right)$ which cuts up $S^{n}$. Denote by $A_{m}$ one such component. Then the diameter of $f\left(A_{m}\right)$ which is connected and which is either in $O_{m}$ or in $E^{1}-O_{m}$ is less than $1 / m$. For each $m$, pick a point $x_{m} \in A_{m}$. Since $S^{n}$ is compact, the sequence $\left\{x_{m}\right\}$ has a limit point. Let $x$ be such a limit point, and set $k=f(x)$. Also let $B_{r}=\{s \mid k-1 / r \leqq$ $s \leqq k+1 / r\}$ and let $C_{r}$ be that component of $f^{-1}\left(B_{r}\right)$ which contains $x$. Then each of the sets $C_{r}$ contains at least one of the sets $A_{m}$. For, there is a number $\delta>0$ for which $|y-x|<\delta$ implies $|f(y)-k|<1 / 2 r$, and there exists $m(\delta)>2 r$ for which $\left|x_{m(\delta)}-x\right|<\delta$. Now $A_{m(\delta)} \subseteq C_{r}$; for, since $\left|x_{m(\delta)}-x\right|<\delta$, the segment of great circle connecting $x$ to $x_{m(\delta)}$ also satisfies this property so that for every point $y$ on this segment $|f(y)-k|<1 / 2 r$ and $x_{m(\delta)} \in C_{r}$. Also for any point $z \in A_{m(\delta)},|f(z)-k| \leqq$ $\left.\left|f(z)-f\left(x_{m(\delta)}\right)\right|+\mid f\left(x_{m(\delta)}\right)\right)-k \mid<1 / 2 r+1 / 2 r=1 / r$. Thus the connected set consisting of the segment and $A_{m(\delta)}$ is all mapped into $B_{r}$, so that $C_{r}$ contains $A_{m(\delta)}$ and hence $C_{r}$ cuts up $S^{n}$ for each $r$.

Now let $C=\bigcap_{r=1}^{\infty} C_{r}$. $C$ is then the intersection of a decreasing sequence of closed, connected sets in a compact space and is thus closed, connected and nonempty. ([3] page 81.) Quite clearly, $x \in C$ and $f(C)=$ $k$. Suppose now that $C$ does not cut up $S^{n}$. Then there is a component, say $D$, of $S^{n}-C$, with measure more than one-half. Let $w \in D$. For all sufficiently large $r, w \notin C_{r}$. Let $D_{r}$ be that component of $S^{n}-C_{r}$ which contains $w .\left\{D_{r}\right\}$ is an increasing sequence of open connected sets. $D=\bigcup_{r=1}^{\infty} D_{r}$ for otherwise there would be a point $v \in D$ not in any $D_{r}$. $\quad D$ being open and connected contains an are joining $w$ to $v$. If $v \notin \cup D_{r}$, there is a first point $u$ along this arc such that $u \notin \cup D_{r}$. But since $u \in D, u \notin \cap C_{r}$ so for some $r, u \notin C_{r}$. For this value of $r$, $u$ and some neighborhood of it are $S^{n}-C_{r}$. Also for some $i>r$, points of this neighborhood are in $D_{i}$, and so must $u$ be. Thus $u \in \cup D_{r}$ and this contradiction establishes that $D=\cup D_{r}$. But now each $D_{r}$ is a component of the complement of $C_{r}$ and each $C_{r}$ contains some $A_{m}$. Hence, since each $A_{m}$ cuts up $S^{n}$, each $D_{r}$ has measure not greater than one-half. However, the expanding union of sets with measure not greater than one-half cannot have measure greater than one-half, so that $D$ has measure no greater than one-half contrary to the hypothesis above, and $C$ does cut up $S^{n}$. This concludes the proof of Theorem 2.

Extensions and related topics. The only property of the real numbers used in the foregoing is that fact that for every $\varepsilon>0$, there exists an open subset of them with the property that every component of the open set and of its complement has diameter less than $\varepsilon$. Thus the reals could be replaced by any (metric) space with this property. Hence, since $E^{1}$ cannot be replaced by $E^{2}$ in Theorem 2, we conclude 
that $E^{2}$ does not have this property. The theorems which follow are easily deducible from this fact.

THEOREM. If $O$ is an open subset of the unit square $I \times I$, then either some component of $O$ or some component of $(I \times I)-O$ contains a pair of points belonging to opposite faces of $I \times I$.

THEOREM. If $f: I \times I \rightarrow E^{1}$ is continuous, there is a connected subset of $I \times I$ which contains a pair of points on opposite faces of $I \times I$ and on which $f$ is a constant.

Theorem. If $f: S^{1} \times S^{1} \rightarrow E^{1}$ is continuous, and if $p: E^{2} \rightarrow S^{1} \times$ $S^{1}$ is the usual projection map of $E^{2}$ as the universal covering space of $S^{1} \times S^{1}$, then there is a connected subset $A$ of $E^{2}$ such that diam $A=\infty$ and $f p \mid A$ is a constant.

THEOREM. If $f: S^{1} \times S^{1} \rightarrow E^{1}$ is continuous, there is a connected subset $B$ of $S^{1} \times S^{1}$ such that $f \mid B$ is a constant and such that $B$ carries a nontrivial one-dimensional Cech cycle of $S^{1} \times S^{1}$.

The proofs of all these theorems are straightforward and are given in the author's dissertation [4].

A different extension is given by the following theorems.

TheoRem. If $n \geqq 2 m+1$ and $f: S^{n} \rightarrow E^{m}$ is continuous, there exists a connected subset of $S^{n}$ which contains a pair of antipodal points and on which $f$ is constant. (This theorem follows easily from Yang [6].)

THEOREM. If $n \leqq 2 m-1, m \geqq 1$, there exists a continuous function $f: S^{n} \rightarrow E^{m}$ such that on no connected subset of $S^{n}$ containing a pair of antipodal points is $f$ a constant.

Proof. Consider the case $n=2 m-1 . S^{2 m-1}=\left\{\bar{x}=\left(x_{1}, x_{2}, \cdots, x_{2 m}\right) \mid\right.$ $\left.\sum\left(x_{i}\right)^{2}=1\right\}$. For $1 \leqq i \leqq m$, define $A_{i}, B_{i}$ and $C_{i}$, by $A_{i}=\left\{\bar{x} \mid x_{2 i-1}=\right.$ $\left.O, x_{2 i} \geqq O\right\}, B_{i}=\left\{\bar{x} \mid x_{2 i}=-x_{2 i-1}, x_{2 i-1} \geqq O\right\}$ and $C_{i}=\left\{\bar{x} \mid x_{2 i}=x_{2 i-1}, x_{2-1} \leqq O\right\}$. Let $D_{i}=A_{i} \cup B_{i} \cup C_{i}, 1 \leqq i \leqq m$. Let $f_{i}: S^{2 m-1} \rightarrow E^{1}$ be given by $f_{i}(y)=d\left(y, D_{i}\right)$. Since every closed connected set containing a pair of antipodal points of $S^{2 m-1}$ intersects $D_{i}$, the points which are connected to their antipodal points by a level set of $f_{i}$ consist of those points for which $x_{i}=x_{i+1}=0$. Thus $f: S^{2 m-1} \rightarrow E^{m}$ given by $f=$ $\left(f_{1}, f_{2}, \cdots, f_{m}\right)$ satisfies the conditions of the theorem. For $n<2 m-$ 1 one can take a great $n$-sphere on the $2 m-1$ sphere and use the restriction of the above example. 
I can give no information in the case $f: S^{2 m} \rightarrow E^{m}, m \geqq 2$.

\section{BIBLIOGRAPHY}

1. P. R. Halmos, Measure Theory, Van Nostrand (1954), page 38.

2. R. D. Johnson, Jr., Bulletin of the American Mathematical Society, v. 60, page 36 (1954), abstract.

3. M. H. A. Newman, Elements of the Topology of Plane Sets of Points (Second edition), Cambridge, 1951.

4. L. M. Sonneborn, Level Sets on Spheres, Ph. D. thesis, California Institute of Technology, 1956.

5. R. L. Wilder, Topology of Manifolds, American Mathematical Society, 1949.

6. C. T. Yang, On Theorems of Borsuk-Ulam, Kakutani-Yamabe-Yujobô and Dyson II, Annals of Mathematics, 62 (1955), 273.

THE UNIVERSITY OF KANSAS 



\section{PACIFIC JOURNAL OF MATHEMATICS}

\section{EDITORS}

\section{RalPh S. Phillips}

Stanford University

Stanford, California

M. G. Arsove

University of Washington

Seattle 5, Washington
J. Dugundji

University of Southern California Los Angeles 7, California

Lowell J. Paige

University of California

Los Angeles 24, California

\section{ASSOCIATE EDITORS}
E. F. BECKENBACH
D. DERRY
H. L. ROYDEN
E. G. STRAUS
T. M. CHERRY
M. OHTSUKA
E. SPANIER
F. WOLF

\section{SUPPORTING INSTITUTIONS}

\author{
UNIVERSITY OF BRITISH COLUMBIA \\ CALIFORNIA INSTITUTE OF TECHNOLOGY \\ UNIVERSITY OF CALIFORNIA \\ MONTANA STATE UNIVERSITY \\ UNIVERSITY OF NEVADA \\ NEW MEXICO STATE UNIVERSITY \\ OREGON STATE UNIVERSITY \\ UNIVERSITY OF OREGON \\ OSAKA UNIVERSITY \\ UNIVERSITY OF SOUTHERN CALIFORNIA
}

STANFORD UNIVERSITY

UNIVERSITY OF TOKYO

UNIVERSITY OF UTAH

WASHINGTON STATE UNIVERSITY

UNIVERSITY OF WASHINGTON

AMERICAN MATHEMATICAL SOCIETY

CALIFORNIA RESEARCH CORPORATION SPACE TECHNOLOGY LABORATORIES

NAVAL ORDNANCE TEST STATION

Printed in Japan by International Academic Printing Co., Ltd., Tokyo Japan 


\section{Pacific Journal of Mathematics}

\section{Vol. 13, No. 1 \\ March, 1963}

Frantz Woodrow Ashley, Jr., A cone of super-(L) functions............. 1

Earl Robert Berkson, Some metrics on the subspaces of a Banach space....

Felix Earl Browder and Walter Strauss, Scattering for non-linear wave

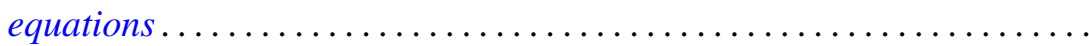

Edmond Darrell Cashwell and C. J. Everett, Formal power series ..........

Frank Sydney Cater, Continuous linear functionals on certain topological

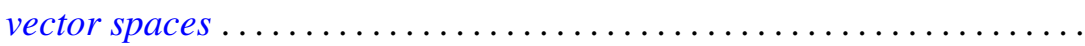

John Douglas Dixon, General group extensions ....................

Robert Pertsch Gilbert, On harmonic functions of four variables with

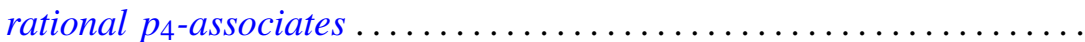

Irving Leonard Glicksberg, On convex hulls of translates ..............

Simon Hellerstein, On a class of meromorphic functions with deficient zeros

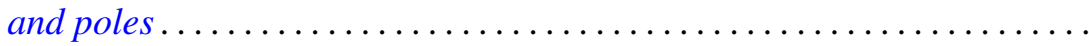

Donald William Kahn, Secondary cohomology operations which extend the

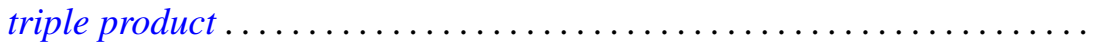

G. K. Leaf, A spectral theory for a class of linear operators .............

R. Sherman Lehman, Algebraic properties of the composition of solutions of partial differential equations ........................... 157

Joseph Lehner, On the generation of discontinuous groups ............. 169

S. P. Lloyd, On certain projections in spaces of continuous functions ...... 171 Fumi-Yuki Maeda, Generalized spectral operators on locally convex spaces ..................................

Donald Vern Meyer, $E^{3}$ modulo a 3-cell

William H. Mills, An application of linear programming to permutation groups.

Richard Scott Pierce, Centers of purity in abelian groups

Christian Pommerenke, On meromorphic starlike functions ...

Zalman Rubinstein, Analytic methods in the study of zeros of

polynomials...

B. N. Sahney, On the Nörlund summability of Fourier series

Tôru Saitô, Regular elements in an ordered semigroup . .

Lee Meyers Sonneborn, Level sets on spheres...........

Charles Andrew Swanson, Asymptotic estimates for limit point

problems .

Lucien Waelbroeck, On the analytic spectrum of Arens . .

Alvin (Murray) White, Singularities of a harmonic function of three

variables given by its series development .............

Kōichi Yamamoto, Decomposition fields of difference sets ...

Chung-Tao Yang, On the action of $\mathrm{SO}(3)$ on a cohomology manifold... 